\title{
GPPS-BJ-2019-0229
}

\section{METHODOLOGY FOR HIGH-ACCURACY INFRARED CALIBRATION IN ENVIRONMENTS WITH THROUGH-WALL HEAT FLUX}

\author{
Mathias Michaud, Francesco Ornano, Nafiz H. K. Chowdhury and Thomas Povey* \\ Oxford Thermofluids Institute, Department of Engineering Science, University of Oxford, Parks Road, \\ Oxford, OX1 3PJ, UK \\ ${ }^{*}$ Corresponding author: thomas.povey@eng.ox.ac.uk
}

\begin{abstract}
This paper describes a new method for accurate in situ infrared (IR) calibration in environments with significant through-wall heat flux and surface temperature nonuniformity. In the context of turbine research environments, conventional approaches for in situ IR calibrations rely on thermocouples embedded in the surface or bonded to the surface using an adhesive layer. A review of the literature points to lack of emphasis on the uncertainty in the calibration arising from the effect of the adhesive substrate and paint on the temperature measured by the thermocouple, namely that under diabatic conditions (i.e. with through-wall heat flux) the measured temperature deviates from the true surface temperature.

We present a systematic study of the sensitivity of the thermocouple temperature to installation conditions seen in typical laboratory IR calibration arrangements, and under realistic conditions of through-wall heat flux. A new technique is proposed that improves the calibration accuracy by reducing the difference between the thermocouple measurement and the external wall temperature seen by the infrared camera. The new technique has the additional advantage of reducing the uncertainty associated with selecting an appropriate pixel in the IR image, by providing a region with greater temperature uniformity especially in environments with significant underlying lateral surface temperature variation. The new approach is experimentally demonstrated and compared to more conventional measurement techniques on a heavily film-cooled nozzle guide vane assembly operated at highly engine-representative conditions. The proposed technique is demonstrated to significantly improve the measurement accuracy for IR in situ calibrations in environment with through-wall heat flux and surface temperature non-uniformity.
\end{abstract}

\section{INTRODUCTION}

As the spatial resolution and internal calibration stability of IR cameras has improved, they have become more popular as research instruments in the gas turbine heat transfer community. It is common practice to calibrate the camera (IR radiometric value) against a region of known temperature (typically by thermocouple measurement) in the field of view (FOV) of the camera. The in situ calibration, whilst typically having the disadvantage of less accurate surface temperature measurement (thermocouple instead of RTD, as it would be common in dedicated calibration instruments), has the significant advantage of directly accounting for poorly characterized target surface emissivity, angle variation and transmissivity of elements in the optical path (windows in pressure vessels typically being the most significant). This is the prime reason for it being the most common method for target surface temperature evaluation (over methods involving separate out-of-experiment calibrations, and associated correction methods). Typical methods for instrumenting the target surface include embedding a thermocouple in the surface, or adhering it to the surface by means of an adhesive layer. Data is acquired simultaneously from both the IR camera and the thermocouple at a fixed location in space (i.e. fixed pixel location for the IR camera), resulting in a direct calibration for the target surface. Any thermal resistance (an adhesive layer, for example) between the thermocouple and the target surface will lead to a measurement error by the thermocouple in situations where there is through-wall heat flux. The presence of an adhesive layer will also cause a lateral temperature disturbance locally near the thermocouple junction. This complicates the formation of an accurate calibration curve, because of the high sensitivity to the selection of the thermocouple junction location (often just a few pixels wide) in a region of high lateral temperature variation. Additional uncertainty in the calibration chain can arise if an adhesive layer forms on the outer face of the thermocouple during its installation, due to the thermal resistance between the thermocouple junction and the surface being visualized. Indeed, a similar effect is often deliberately introduced in the form of a thin paint layer, in an effort to render the entire surface of uniform emissivity. 


\section{Literature relating to practice for IR calibration experiments}

In the case of highly non-uniform wall heat transfer and surface temperature gradients (typical of, for instance, film cooling environments), high spatial resolution is required to fully characterize the complex thermal field. IR thermography is a perfect candidate for this type of applications as it offers both high spatial and thermal resolution. The accuracy of quantitative IR measurements relies on calibration experiments performed to correlate the radiometric signal from the camera to a known temperature. In turbine research environments (namely, represented by wind tunnels operating at either low- or high-speed conditions, with surface temperature variations), bespoke in situ experiments are required to calibrate the IR camera at the nominal test conditions. In situ tests are performed to correct for poorly characterized target surface emissivity, view angle variation, and transmissivity of elements in the optical path.

It is common practice when carrying out in situ calibration tests to compare the radiometric data to a thermocouple temperature at the thermocouple location in the IR image. A number of thermocouples are therefore embedded or bonded to the surface with an adhesive layer, and the temperature measured by the thermocouples used for calibration should cover the complete temperature range of interest.

Martiny et al. (1996) presented an in situ calibration technique for quantitative infrared measurements. The raw detector signal from the infrared camera was compared to the temperatures measured with thermocouples. The function between raw detector value and object temperature was cast in the form of the Plank equation. The latter included three parameters, which were used for a best-fit approximation. The resulting non-linear system of equations was solved numerically and the calculated parameters were used to estimate the corrected temperature of the body.

Schulz (2000) presented a quantitative infrared approach applied to film cooling research. Their work described the theoretical aspects and methods related to in situ calibrations. Examples of film cooling investigations using infrared thermography were also presented. In their experiments, a number of thermocouples were embedded in the surface and used to directly calibrate the radiometric signal from a single IR camera.

Ochs et al. (2009) proposed a technique to overcome the issue related to thermocouples being located in regions of high surface temperature gradients. The technique allows for data extrapolation with improved accuracy when calibrating the radiometric data to the thermocouple temperature. The approach was based on the determination of three of four calibration parameters by a pre-calibration run and a subsequent in situ calibration to estimate the value of the fourth parameter. They claimed that fewer thermocouples were necessary for the in situ calibration, and that thermocouples did not have to cover the entire temperature operating range (making therefore possible to discard the thermocouples located in regions of high temperature gradients). The method was applied to the thermal investigation of transonic trailing edge cooling and film cooling of a flat plate.
Despite the conspicuous number of papers on the application of IR thermography on film cooling research, we can find no mention of the effect of the calibration arrangement on the accuracy of surface temperature measurements. As it will be shown later, there is a strong sensitivity of the primary sources of error in the calibration to variations in the parameters involved in the measurement setup. In fact, various parameters such as adhesive/paint thickness and thermal conductivity substantially affect the temperature measured by the thermocouple and therefore can lead to increased overall uncertainty in the calibration process.

This paper presents a parametric study on the influence of the measurement layout on the temperature measured by the thermocouple. The influence of a number of physical parameters is assessed in the context of their effect on the difference between thermocouple temperature measurement used for calibration and the temperature of the surface seen by the IR camera. A new instrumentation methodology is proposed which has significant advantages over more conventional methods, in that it mitigates the primary sources of error. The method offers a low thermocouple measurement error in the presence of through-wall heat flux and a high accuracy in localizing the reference thermocouple in regions of high surface temperature gradients. In the proposed layout, a low conductivity substrate is implemented to locally minimize the through-wall heat flux, and a high thermal conductivity layer is bonded to a thermocouple by means of thermally conductive paste. The high conductivity layer is designed to operate at low values of the Biot number, namely that uniform spatial temperature is reached during the test. An area including several pixels at uniform temperature is therefore available in the IR image, leading to substantially reduced error when calibrating the radiometric signal to the true wall temperature measured by the thermocouple.

It is hoped that results from this paper will be useful to researchers to understand the influence of the physical parameters involved in the calibration experiments, and to quantify the systematic primary error introduced by the system layout in the surface temperature measurement.

\section{ASSESSMENT OF ERRORS ASSOCIATED WITH CONVENTIONAL MEASUREMENT SYSTEMS}

We now consider typical errors in the most common thermocouple temperature measurement and calibration systems. This discussion is progressed under two headings: sensitivity of surface temperature measurement to adhesive and paint layers; and sensitivity of surface temperature measurement to pixel selection in regions of high temperature gradient.

\section{Sensitivity of surface temperature measurement to adhesive and paints layers}

In this section, we assess the sensitivity of the surface temperature measurement to the structure of the surface measurement system. In particular, we consider the effect of adhesive and paint layers on the on the temperature measured by the thermocouple. We use a one-dimensional steady-state heat transfer model to consider the impact of thermal 
conductivities and thickness of such layers in a number of common surface measurement systems.

Figure 1 shows three conventional surface measurement systems used for in-situ IR calibration. We refer to these as reference systems 1 to 3. The systems are: (1) thermocouple over adhesive; (2) over-painted thermocouple over adhesive; (3) thermocouple between adhesive layers. Reference system 1 is representative of a typical foil thermocouple (used to minimise surface aero-thermal disruption) adhered directly to a surface. Reference system 2 is a modified version of system 1 , in which the foil thermocouple is additionally overpainted to render the surface of uniform emissivity. Reference system 3 represents a foil thermocouple that is adhered between adhesive layers (sometimes used either for robustness, or arising due to unintentional over-coating with glue when adhering with resin-based glue).

In the analysis of these systems, the wall is subject to a (hot) external flow at total temperature $T_{0, \text { ext }}$ with heat transfer coefficient $h_{\text {ext }}$. The internal surface is subject to a flow of total temperature $T_{0 \text {,int }}$ with heat transfer coefficient $h_{\text {int }}$. The particular boundary conditions are summarised in Table 1, and are representative of typical laboratory turbine testing conditions with Reynolds and Mach numbers matched to engine conditions, but with temperature ratio $T_{0, \text { ext }} / T_{0 . \text { int }}$ lower than the engine situation (nominal temperature ratio). The wall properties are summarised in Table 2, and correspond to those of Inconel, which is a common material for high-pressure turbine components.

In the analysis of these systems, we differentiate between three types of measurement error: (1) error between the thermocouple measurement and the surface temperature directly above the thermocouple junction; (2) error between the thermocouple measurement and the surface temperature near the thermocouple junction; (3) the error between the thermocouple measurement and the true undisturbed wall temperature (without the presence of the thermocouple, or any associated surface treatment, for example thin over paint layers). Errors of type 1 are the direct error arising in the calibration process when selecting a pixel directly above the thermocouple junction to compare against the thermocouple data. This error is intrinsic in all calibration processes and is difficult to remove. Errors of type 2 arise when there is lateral surface temperature variation, when either the selected pixel in the IR image is not the one directly above the thermocouple junction, or where there is a requirement to average a region in the vicinity of the thermocouple. Errors of type 3 (between the thermocouple measurement and the true undisturbed wall temperature) are most relevant for thermal analysis of a particular component, when the effect of all instrumentation (including thin paint layers) must be accounted for.

These errors, presented in a form normalised by the total temperature difference between the streams, are defined by equations 1 to 3 . The temperatures used in these definitions (except for the true undisturbed wall temperature) are defined in Figure 2. In this paper we are concerned with measurement errors of type 1 and $2\left(\beta_{1}\right.$ and $\left.\beta_{2}\right)$, which lead directly to uncertainty in the IR surface calibration (IR radiometric value for a given measured wall temperature). Where the wall treatment (over-paint layer) is known, error type $3\left(\beta_{3}\right)$ is relatively easy to deal with in back-analysis of a test part.

Table 1: Wall boundary conditions.

\begin{tabular}{lcc}
\hline \hline Boundary condition & Symbol & Value \\
\hline External HTC & $h_{\text {ext }}$ & $1500 \mathrm{~W} \mathrm{~m}^{-2} \mathrm{~K}^{-1}$ \\
Internal HTC & $h_{\text {int }}$ & $2000 \mathrm{~W} \mathrm{~m}^{-2} \mathrm{~K}^{-1}$ \\
External flow total temperature & $T_{0, \text { ext }}$ & $350 \mathrm{~K}$ \\
Internal flow total temperature & $T_{0, \text { int }}$ & $293 \mathrm{~K}$ \\
\hline \hline
\end{tabular}

Table 2: Wall thermal properties.

\begin{tabular}{lcc}
\hline \hline Property & Symbol & Value \\
\hline Wall thickness & $t_{\mathrm{w}}$ & $1.50 \mathrm{~mm}$ \\
Wall conductivity & $k_{\mathrm{w}}$ & $11.7 \mathrm{~W} \mathrm{~m}^{-1} \mathrm{~K}^{-1}$ \\
\hline \hline
\end{tabular}
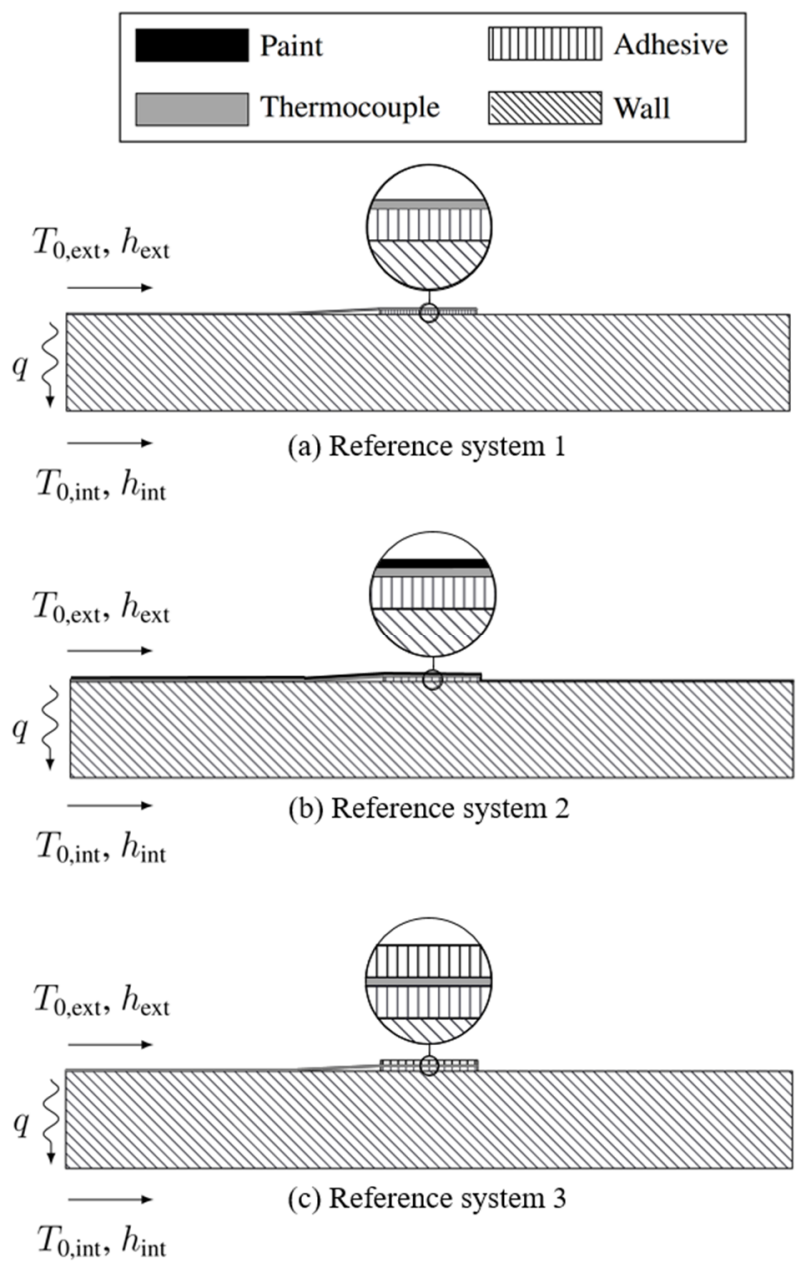

Figure 1: Diagrams of the reference systems (scale 4.5:1): (a) thermocouple over adhesive; (b) over-painted thermocouple over adhesive; (c) thermocouple between adhesive layers. 


$$
\begin{gathered}
\beta_{1}=\frac{T_{\mathrm{w}, \text { measured by TC }}-T_{\mathrm{w}, \text { over TC }}}{T_{0, \text { ext }}-T_{0, \text { int }}} \\
\beta_{2}=\frac{T_{\mathrm{w}, \text { measured by TC }}-T_{\mathrm{w}, \text { next to TC }}}{T_{0, \text { ext }}-T_{0, \text { int }}} \\
\beta_{3}=\frac{T_{\mathrm{w}, \text { measured by TC }}-T_{\mathrm{w}, \text { true }}}{T_{0, \text { ext }}-T_{0, \text { int }}}
\end{gathered}
$$

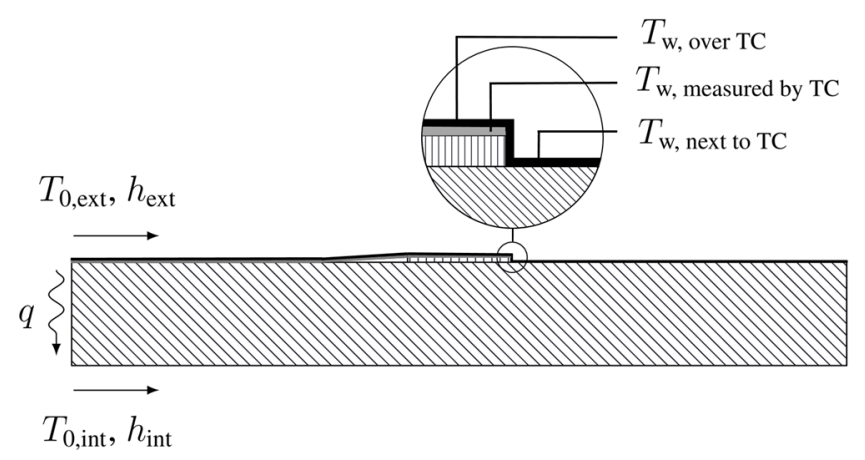

Figure 2: Diagram of gauge structure showing definitions of the temperature measurements.

\section{Reference system 1: thermocouple over adhesive}

Since the thermocouple junction in reference system 1 (Figure 1a) is exposed (not covered by paint or adhesive), the measured temperature by the thermocouple is the same as the temperature directly above the thermocouple junction. Therefore, reference system 1 is not affected by errors of type 1 ; i.e. $\beta_{1}$ is zero. We note for completeness, however, that systems of this type are rarely seen in practice, due to extreme variation in the surface emissivity when the overpaint layer of reference system 2 is omitted (most test surfaces have emissivity close to unity, but untreated foil thermocouples are highly reflective with an emissivity closer to zero).

In the presence of through-wall heat flux, the adhesive layer underneath the thermocouple causes errors of type 2 $\left(\beta_{2}\right)$. This error becomes relevant in almost all practical situations (see later example) where it is necessary to average a number of pixels in the vicinity of the thermocouple because of the difficulty of identifying the thermocouple junction in regions of high surface temperature gradient.

Figure 3 presents $\beta_{2}$ as a function of the adhesive thermal conductivity and thickness underneath the thermocouple for reference system 1 . It is worth nothing that only a fraction of this error that is passed into the final calibration uncertainty, but in many practical situations this fraction may either be high, or at the very least difficult to assess. The discussion is taken up in an example later in this paper.

A foil thermocouple with a standard epoxy bonding layer would typically have an adhesive layer thickness of 40.0 $80.0 \mu \mathrm{m}$. Although epoxy adhesives offer a high bonding strength, typical thermal conductivities of these adhesives are very low: typically in the range $0.100-0.200 \mathrm{~W} \mathrm{~m}^{-1} \mathrm{~K}^{-1}$ (Lee and Neville, 1967). This range of values is marked in the figure as (a). For the through-wall heat flux conditions considered (Table 1), the error for the worst-case estimated values of thermal conductivity and layer thickness can be up to $20.0 \%$ of the total temperature difference between the streams. Even at the best-case end of the estimated range errors of $7.0 \%$ are likely. It is clear that using instrumentation systems of this type it is necessary to be extremely careful with installation effects. Even so, using such systems for high-accuracy calibration systems would appear to be a risky proposition.

An alternative option would be to use a thermally conductive epoxy, which has a thermal conductivity in the range $1.00-2.00 \mathrm{~W} \mathrm{~m}^{-1} \mathrm{~K}^{-1}$. This situation is marked as (b) in the figure. Even for thermally conductive epoxy, the estimated range of errors is between 0.80 and $3.00 \%$ of the total temperature difference between the streams. Although the use of high conductivity epoxy partially mitigates the errors arising in high through-wall heat flux situations, errors can still be high in the context of high accuracy calibration requirements.

An improved option might be to use a high thermal conductivity (and low electrical conductivity) adhesive. Such adhesives would typically also have a bonding layer thickness in the range $40.0-80.0 \mu \mathrm{m}$, with a thermal conductivity in the range $3.50-5.00 \mathrm{~W} \mathrm{~m}^{-1} \mathrm{~K}^{-1}$. This range of values is marked in the figure as (c). For best-case estimated values of thermal conductivity and layer thickness, the error is estimated to be at least $0.30 \%$ of the total temperature difference between the streams. The measurement error can be up to $0.90 \%$ for the worst case. However, the typical tensile strength of high thermal conductivity adhesives is considerably lower than standard epoxy resins (1.00-2.00 $\mathrm{MPa}$, versus more than 20.0 MPa for more common epoxy resins) and this is a serious consideration in high-speed flow environments.

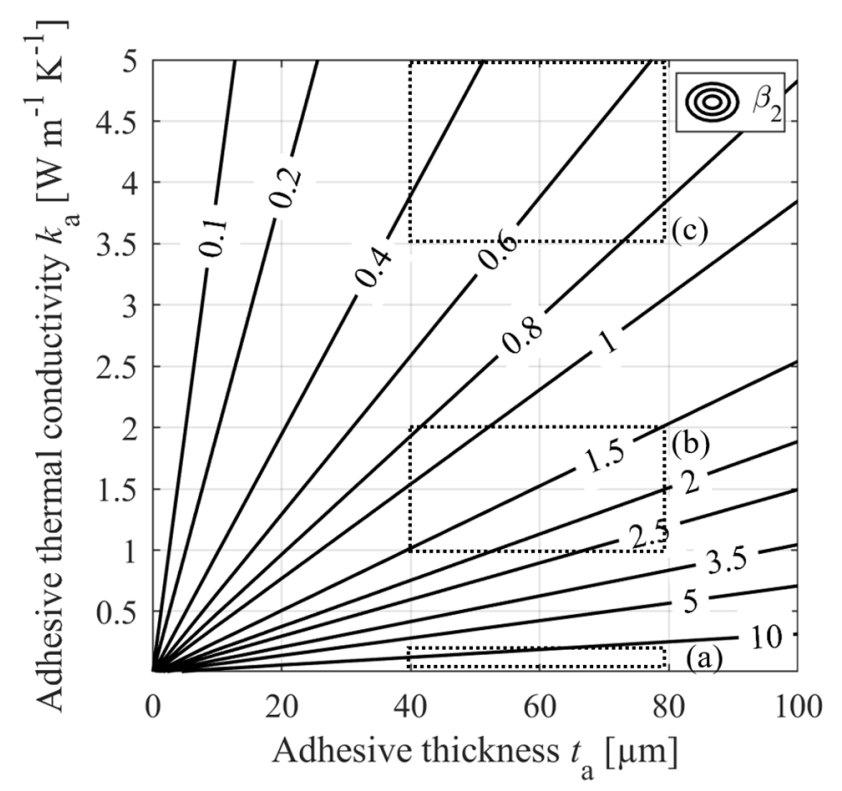

Figure 3: Analysis of reference system 1, showing $\beta_{2}$ (in $\%)$ as a function of adhesive thickness and conductivity. 
Reference system 2: over-painted thermocouple over adhesive

It is a common practice in IR thermography to spray a thin layer of paint over the entire target surface (including the reference thermocouple) to render the surface of uniform emissivity. This situation is shown in Figure 1b. In the presence of through-wall heat flux, the over-paint layer acts as an additional thermal resistance, and raises (for higher mainstream gas temperature) the surface temperature (visualised by the IR camera) with respect to the thermocouple measurement. This leads to errors of type 1 . The effect is rather different to that of underside adhesive, which raises both the thermocouple temperature and the wall temperature above it, leading to errors of type 2 where pixels near to, but not directly above the thermocouple are considered as part of the calibration process. Because nearby pixels (not over the adhesive layer on which the thermocouple is mounted) would tend to register lower temperature in an IR image, the type 2 error is generally of opposite sign to the type 1 error.

Matt black acrylic paint is commonly used in IR thermography, and has a thermal conductivity in the range $0.500-1.50 \mathrm{~W} \mathrm{~m}^{-1} \mathrm{~K}^{-1}$ (Raghu and Philip, 2006). With wellcontrolled spraying, a layer thickness as low as $10.0 \mu \mathrm{m}$ is possible. Figure 4 presents errors of type 1 and $2\left(\beta_{1}\right.$ and $\left.\beta_{2}\right)$ as a function of the paint thermal conductivity and layer thickness for an adhesive with $40.0 \mu \mathrm{m}$ thickness and thermal conductivity of $5.00 \mathrm{~W} \mathrm{~m} \mathrm{~K}^{-1}$. This adhesive layer represents the thinnest possible layer of high thermal conductivity adhesive estimated for reference system 1 , corresponding to the lowest possible estimated values of $\beta_{1}$ and $\beta_{2}$ ( 0.00 and $0.30 \%$ respectively). These errors are the starting point for our current calculation, and we recall the effects lead to errors with opposite sign.

Figure 4 shows type 1 and type 2 errors, $\beta_{1}$ and $\beta_{2}$, as a function of paint thickness and conductivity. So far as type 1 errors are concerned, in the presence of through-wall heat flux the temperature difference between the thermocouple and the surface directly above it increases with increasing paint thickness and with decreasing paint thermal conductivity, leading to an increase in the absolute magnitude of the error. The effect is due to the increase in wall resistance between the thermocouple junction and the surface being visualised. So far as type 2 errors are concerned, the effect is broadly similar in nature and magnitude to the type 1 error, but non-linearity in the additive effect of the wall resistances leads to slightly different trends. We observe that for the nominal paint thickness of $10.0 \mu \mathrm{m}$ at the best-case estimated value of paint thermal conductivity, $\beta_{1}$ and $\beta_{2}$ are respectively -0.50 and $-0.20 \%$. As for the worst-case estimate of the paint thermal conductivity, $\beta_{1}$ and $\beta_{2}$ are respectively -1.50 and $-1.20 \%$. This demonstrates that adding paint over a reference measurement system (almost universal in practice, for reasons discussed above) can lead to significant errors. The direct calibration errors $\left(\beta_{1}\right)$ in particular can be extremely difficult to remove.

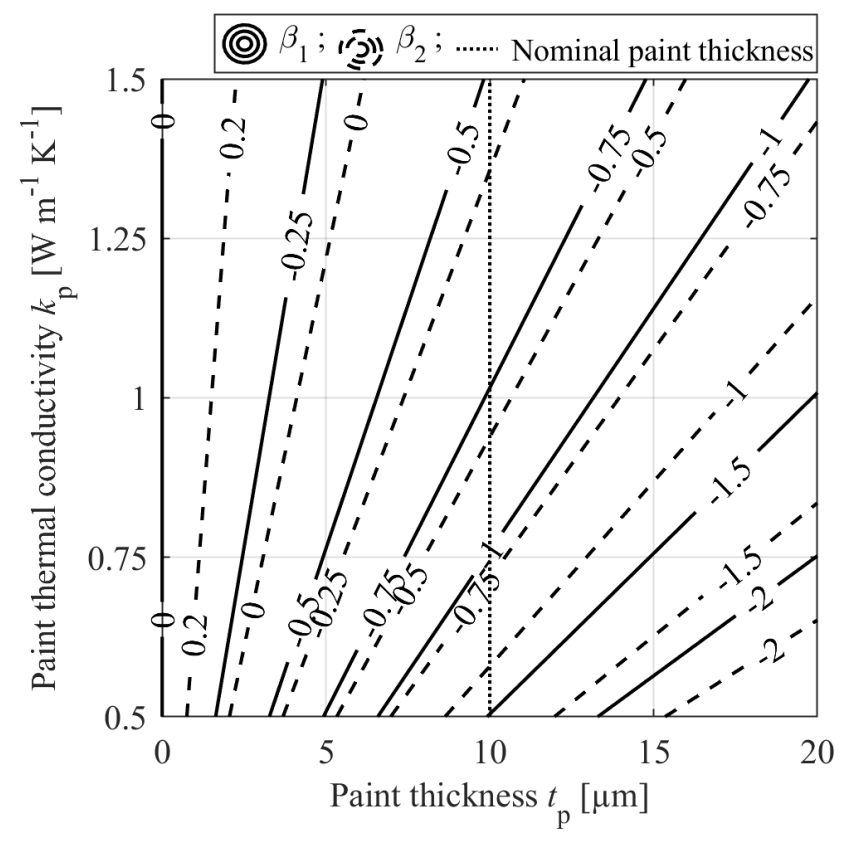

Figure 4: Analysis of reference system 2, showing $\beta_{1}$ and $\beta_{2}$ (in \%) as a function of paint thickness and conductivity, for an adhesive layer with thickness $\mathbf{4 0 . 0}$ $\mu \mathrm{m}$ and thermal conductivity $5.00 \mathrm{~W} \mathrm{~m}^{-1} \mathrm{~K}^{-1}$.

\section{Reference system 3: thermocouple between adhesive layers}

During the thermocouple installation a layer of adhesive can form over the thermocouple junction, adding further thermal resistance. Figure 5 and Figure 6 show the contours of $\beta_{1}$ and $\beta_{2}$, respectively, as a function of the thickness and thermal conductivity of the adhesive over the thermocouple, for the case of a $50.0 \mu \mathrm{m}$ thick layer of the same adhesive underneath the thermocouple.

Adhesive over the thermocouple has the effect of introducing error between the local (directly above the thermocouple) external wall temperature and the thermocouple temperature, and is a cause of $\beta_{1}$ error. Adhesive under the thermocouple is unimportant so far as $\beta_{1}$ error is concerned, but is a source of $\beta_{2}$ error.

So far as $\beta_{2}$ errors are concerned, in the presence of through-wall heat flux, adhesive under the thermocouple tends to drive up the thermocouple temperature with respect to a point unaffected by the adhesive next to the thermocouple. Adhesive over the thermocouple tends to drive the thermocouple temperature down with respect to a nearby region of wall. The effects are therefore in an interesting competition, and can potentially cancel each other out.

A typical adhesive layer thickness over the thermocouple can be up to $20.0 \mu \mathrm{m}$. For standard epoxy adhesives (region marked as (a) in Figure 5), the direct calibration error $\beta_{1}$ can be as large as $-10.00 \%$ for the worst-case estimated thermal conductivity and thickness of the adhesive layer over the thermocouple. For thermally conductive epoxy (marked as (b) in Figure 5), $\beta_{1}$ can be as large as $-1.50 \%$ as a worst case. As for high thermal conductivity adhesive (marked as 
(c) in Figure 5), $\beta_{1}$ can reach $-0.45 \%$ for the worst case. It is clear that during thermocouple installation it is necessary to avoid the migration of adhesive over the thermocouple junction, or significant direct errors in the calibration can arise.

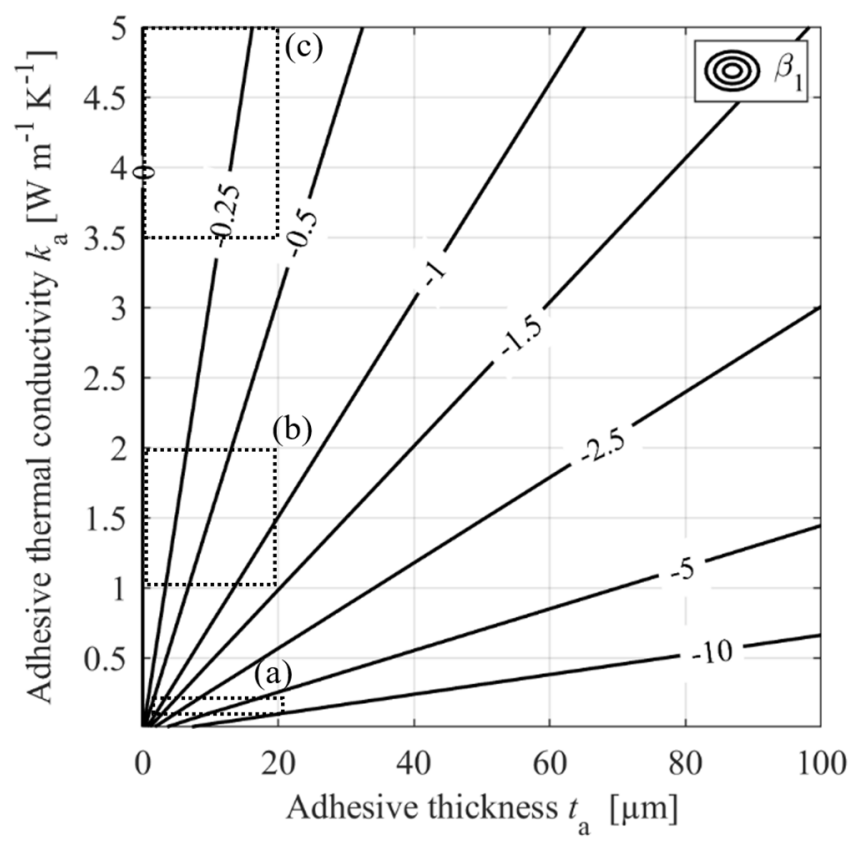

Figure 5: Analysis of reference system 3 , showing $\beta_{1}$ as a function of adhesive thickness and conductivity over the thermocouple, for fixed adhesive layer thickness of $50.0 \mu \mathrm{m}$ under the thermocouple.

Concerning $\beta_{2}$, the smallest error encountered with standard epoxy (region marked as (a) in Figure 6) is $5.00 \%$ for the best-case estimated thermal conductivity and thickness of the adhesive layer over the thermocouple. For thermally conductive epoxy (marked as (b) in Figure 6), $\beta_{2}$ can be as small as $0.64 \%$ as a best case. As for high thermal conductivity adhesive (marked as (c) in Figure 6), $\beta_{2}$ can reach $0.25 \%$ for the best case. For a given adhesive thermal conductivity, the worst-case scenario corresponds to having no adhesive over the thermocouple (i.e. reference system 1 with the same adhesive layer thickness underneath the thermocouple). This shows that having a layer of adhesive over the thermocouple thinner than the layer underneath is beneficial as far as $\beta_{2}$ is concerned (error reduced compared to reference system 1). However, this is very hard to achieve in practice as it is very difficult to control the thicknesses of both layers adhesives.

We numerically assessed the dependency of the thermocouple temperature on the measurement layout and physical properties of the adhesive used. We observed that the measurement error strongly depends on the location, thickness and thermal conductivity of the adhesive layer in the presence of through-wall heat flux. In practice, poorly characterized adhesive/paint thermal properties and thicknesses could lead to significantly large measurement errors and increased overall calibration uncertainty.

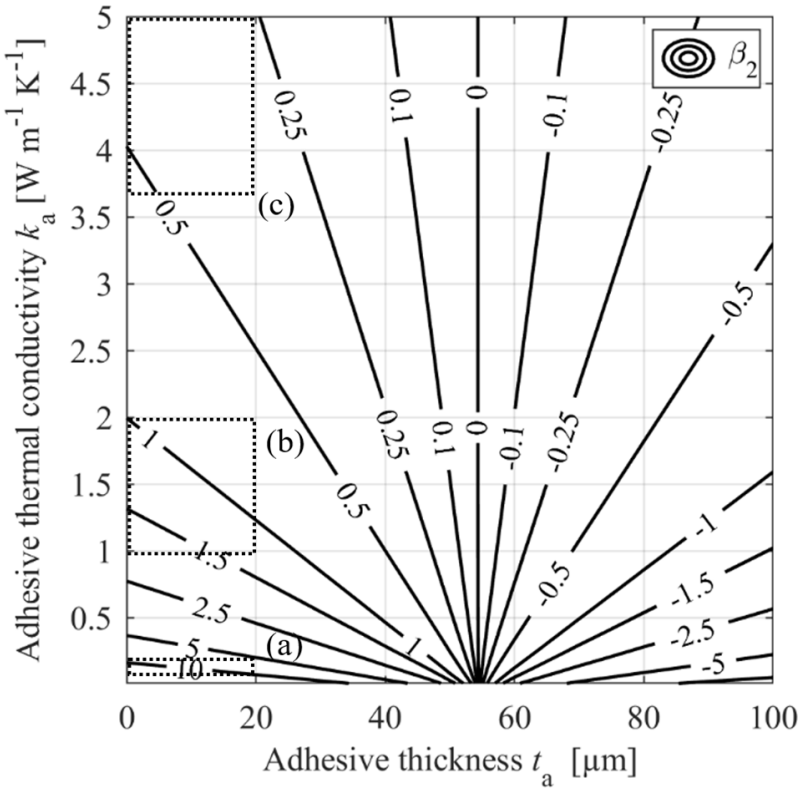

Figure 6: Analysis of reference system 3 , showing $\beta_{2}$ as a function of adhesive thickness and conductivity over the thermocouple, for fixed adhesive layer thickness of $50.0 \mu \mathrm{m}$ under the thermocouple.

Sensitivity of surface temperature measurement to pixel selection in regions of high temperature gradient

To calibrate IR camera output to a measured wall temperature, radiometric values are recorded against thermocouple readings. In this process it is required that the thermocouple reading is as representative as possible of the local surface temperature. If the region of the thermocouple is sufficiently isothermal, the temperature directly above the thermocouple location can be taken. Errors for this situation have been discussed. Where the surface is non-isothermalon account of disruption by the thermocouple system for example-the error associated with region selection must also be considered. In this case, two errors must be considered: the error between the thermocouple and wall temperature directly above the thermocouple $\left(\beta_{1}\right)$; the error between the thermocouple and the wall temperature local to but not directly above the thermocouple $\left(\beta_{2}\right)$. These errors have been considered separately in the preceding sections.

When IR images are taken, it is typical to select a region of several pixels around the thermocouple junction location to establish an area-averaged radiometric value on the target surface. Moderate lateral temperature gradients are possible because of the nature of the components being studied, as shown, for example in the results of Kirollos et al. (2017), which are reproduced in Figure 7. The figure shows a nozzle guide vane non-dimensional surface temperature presented as overall cooling effectiveness $\theta$ (the complete definition of $\theta$ is described in Kirollos et al., 2017). The part was fully cooled (both external film and internal convective cooling) and was operated at conditions non-dimensionally representative of an engine. In these demonstration measurements, a thin foil K-type thermocouple was used (indicated in Figure 7) to calibrate IR data. The thermocouple installation scheme was like reference system 2 (Figure 1b): over-painted thermocouple over adhesive. 


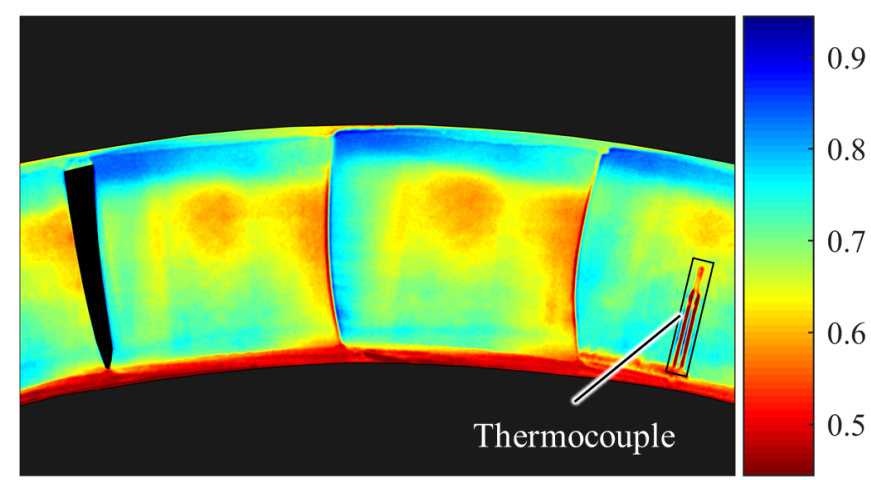

Figure 7: Example of overall cooling effectiveness $(\boldsymbol{\theta})$ measurements (Kirollos et al., 2017) on engine components, showing high lateral temperature variation.

The thermocouple wires are visible in Figure 7 because of the change in surface temperature due to the variation in thermal resistance with respect to the undisturbed surface. This primarily results from a thin layer of epoxy under the leads.

A detail view of the results of Figure 7 is presented in Figure $8 \mathrm{a}$ in terms of normalized temperature $\phi$ in the vicinity of the thermocouple. Here $\phi$ is defined by

$$
\phi=\frac{T_{0, \text { ext }}-T_{\mathrm{w}, \mathrm{bb}}}{T_{0, \text { ext }}-T_{0, \mathrm{int}}}
$$

where $T_{\mathrm{w}, \mathrm{bb}}$ is the equivalent black body temperature of the target surface recorded by the IR camera, and $T_{0 \text {,ext }}$ and $T_{0 \text {,int }}$ are the mainstream total temperatures respectively (defined at vane inlet and the vane coolant plenum inlet).

It is clear that the moderate lateral temperature gradients on the vane surface are compounded by high temperature gradients local to the thermocouple, caused by the disturbance of the thermocouple construction.

This is emphasized by Figure $8 \mathrm{~b}$, which shows the lateral distribution of normalized temperature along the line AA' through the thermocouple junction. Individual points represent single pixels in the IR image. Significant lateral temperature variation is apparent. This situation is very common even with the most careful instrumentation scheme. In this example it is clear that two sources of error must be considered. To reiterate: we must consider the error between the thermocouple measurement and wall temperature directly above the thermocouple junction $\beta_{1}$ (directly relevant to the calibration error); we must consider the error between the thermocouple measurement and the wall temperature local to but not directly above the thermocouple junction $\beta_{2}$ on account of the averaging/region-selection problem that this example exemplifies. These sources of error can be identified in Figure $8 b$. Firstly, we observe that the thermocouple junction takes a low value of the non-dimensional temperature compared to the region in the vicinity $(\phi=$ 0.64). In fact, the structure of the thermocouple determines a change in temperature (due to flow disruption and change in thermal resistance due to adhesive underneath). Secondly, there is a significant lateral temperature variation in the region around the thermocouple (Figure 8a). This primarily affects the error $\beta_{2}$. The effect is visible in Figure $8 \mathrm{~b}$, where the lateral temperature distribution along line AA' passing through the thermocouple junction is plotted. The nondimensional temperature $\phi$ takes a value of around 0.64 at the junction location and rapidly increases to the level corresponding to the undisturbed surface $(\phi=0.69-0.71)$.

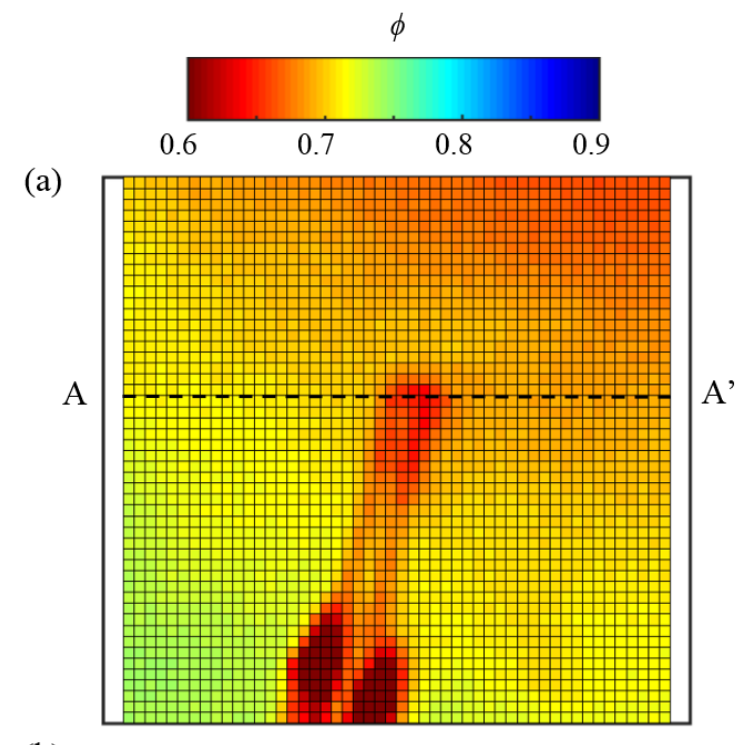

(b) $\quad \phi$ along $\mathrm{AA}^{\prime}$ for conventional system

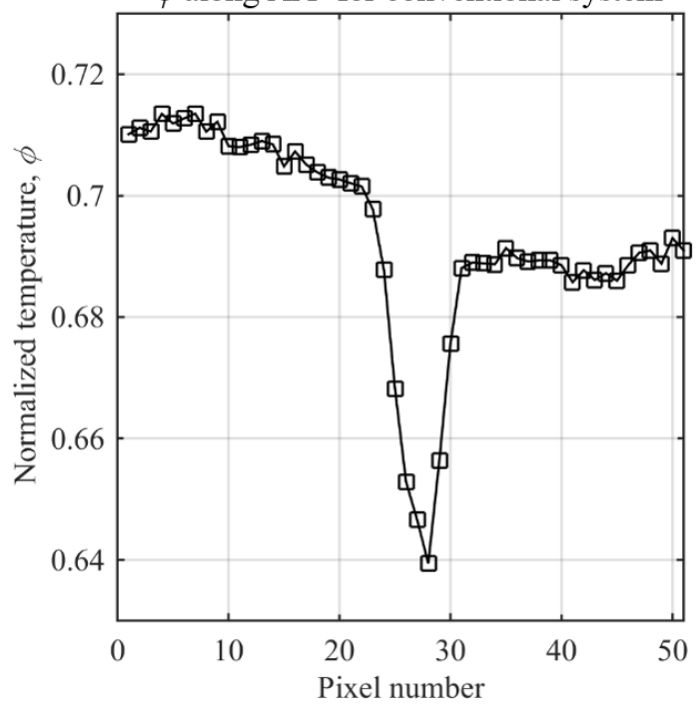

Figure 8: Conventional calibration system (reference system 2): (a) surface plot of $\phi$ over a 50-by-50 pixel area; (b) line-plot of $\phi$ (along $A^{\prime}$ ) through the thermocouple junction.

\section{PROPOSAL FOR AN IMPROVED MEASUREMENT SYSTEM}

We now propose an improved measurement and calibration system (for in situ IR calibration) optimised to reduce measurement error in environments with significant throughwall heat flux and/or lateral temperature gradient. The proposed system is shown in cross-section in Figure 9. We refer to this as reference system 4.

In the assembly, a thin-foil K-type thermocouple is bonded between a disk of copper (high thermal conductivity) and a polyimide film (low thermal conductivity). The 
thermocouple is in direct contact with the bottom face of the copper disk and a layer of thermal paste is applied under the thermocouple and on its sides to adhere it to the copper disk. The copper disk is held in place using an epoxy adhesive and the whole assembly is arranged flush with the target surface to minimize boundary layer disruption. A layer of matt black paint is applied over the calibration and the target surfaces to render them of uniform and equal emissivity. Figure 10 is a photograph (plan view) of the system assembled on the suction side of a HPNGV before over-painting. The cavity into which the assembly was assembled was machined using a $6 \mathrm{~mm}$ diameter flat-bottom cutter to a depth of $0.7 \mathrm{~mm}$. The copper disk is $4 \mathrm{~mm}$ in diameter and is $0.5 \mathrm{~mm}$ thick. Table 3 lists the thickness and thermal conductivity of each element in the assembly.

The proposed measurement system offers several advantages over conventional approaches, specifically:

(1) the low thermal conductivity of the polyimide layer reduces the through-wall heat flux, and in combination with the copper layer, reduces the temperature difference between the thermocouple junction and external surface. This reduces the error between the thermocouple and the wall temperature directly above the thermocouple $\left(\beta_{1}\right)$.

(2) we create a region in the IR camera field of view which is both larger and more isothermal than is typically provided on the target surface with a conventional application of the thermocouple, thus reducing errors associated with thermocouple localized in regions with high temperature variation $\left(\beta_{2}\right)$. In this arrangement the error between the thermocouple and the wall temperature local to but not directly above the thermocouple junction is no longer relevant, because we thermally isolate the calibration patch from the local environment.

(3) the thermocouple junction is protected from the testing environment, allowing the measurement system to be used in high-speed flows.

\begin{tabular}{|c|c|c|}
\hline & Paint & VIIIIIIIA Copper disk \\
\hline & Thermocouple & 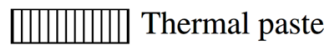 \\
\hline :!:!:! & Epoxy & Polyimide film \\
\hline & Wall & \\
\hline
\end{tabular}

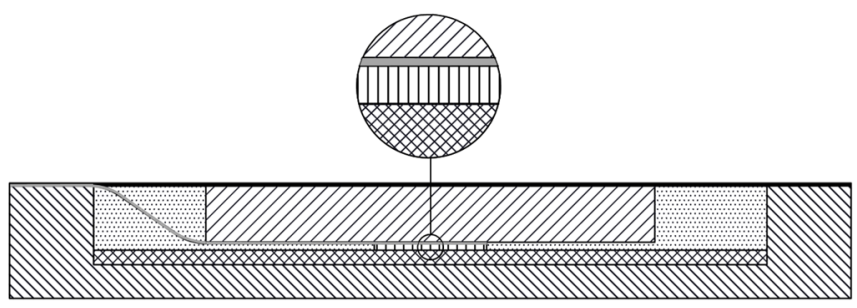

Figure 9: Cross-section of the proposed measurement system (scale 4.5:1): reference system 4.

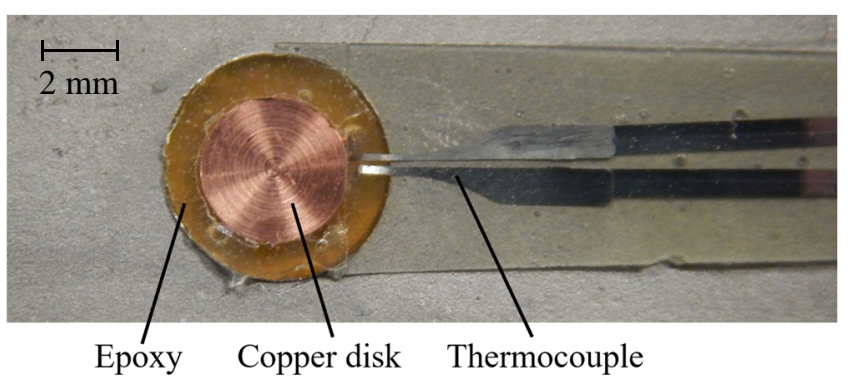

Figure 10: Photograph of the proposed measurement system without paint.

Table 3 - Properties of the elements in the assembly.

\begin{tabular}{lcc}
\hline \hline Element & $\begin{array}{c}\text { Nominal } \\
\text { thickness }\end{array}$ & Thermal conductivity \\
\hline Paint & $10.0 \mu \mathrm{m}$ & $0.500-1.50 \mathrm{~W} \mathrm{~m}^{-1} \mathrm{~K}^{-1}$ \\
Copper disk & $500 \mu \mathrm{m}$ & $386 \mathrm{~W} \mathrm{~m}^{-1} \mathrm{~K}^{-1}$ \\
Thermocouple & $12.3 \mu \mathrm{m}$ & $11.3 \mathrm{~W} \mathrm{~m}^{-1} \mathrm{~K}^{-1}$ \\
Thermal paste & $63.7 \mu \mathrm{m}$ & $3.40 \mathrm{~W} \mathrm{~m}^{-1} \mathrm{~K}^{-1}$ \\
Polyimide film & $124 \mu \mathrm{m}$ & $0.120 \mathrm{~W} \mathrm{~m}^{-1} \mathrm{~K}^{-1}$ \\
\hline \hline
\end{tabular}

\section{DEMONSTRATION OF THE PROPOSED MEASUREMENT SYSTEM}

In this section, we demonstrate how the proposed measurement system (reference system 4) provides a large isothermal region in the FOV of the IR camera, while maintaining a low measurement error of type $1\left(\beta_{1}\right)$ and completely removing error of type $2\left(\beta_{2}\right)$.

To assess the errors in reference system 4 , we created a one-dimensional steady-state heat transfer model of the system shown in Figure 9. The main source of error in the assembly of reference system 4 is the low thermal conductivity of the matt black paint layer applied over the copper disk, which has a range $0.500-1.50 \mathrm{~W} \mathrm{~m}^{-1} \mathrm{~K}^{-1}$ (Raghu and Philip, 2006). Figure 11 presents the contours of $\beta_{1}$, here defined as the error between the thermocouple measurement and the temperature of the painted external surface of the copper disk, as a function of the paint thermal conductivity and the thickness of its layer.

For a nominal paint layer thickness of $10.0 \mu \mathrm{m}$ and the best-case estimated value of paint thermal conductivity, $\beta_{1}$ is at least $0.32 \%$ of the total temperature difference between the streams. As for the worst-case estimated value of paint thermal conductivity, $\beta_{1}$ remains below $0.85 \%$. With reference system 4 , we have taken down the possible range of $\beta_{1}$ of $0.50-1.50 \%$, which is the best that a typical overpainted measurement system like reference system 2 can achieve, to a range of $\beta_{1}$ of $0.32-0.85 \%$. This improvement is possible by thermally insulating the measurement system with only a thin layer of polyimide film to significantly 
reduce the through-wall heat flux and the measurement errors arising from its presence (i.e. the difference between the thermocouple measurement and the external surface temperature seen by the IR camera). As for $\beta_{2}$, reference system 4 completely removes it because the measurement system is laterally isothermal and is separated from its surrounding.

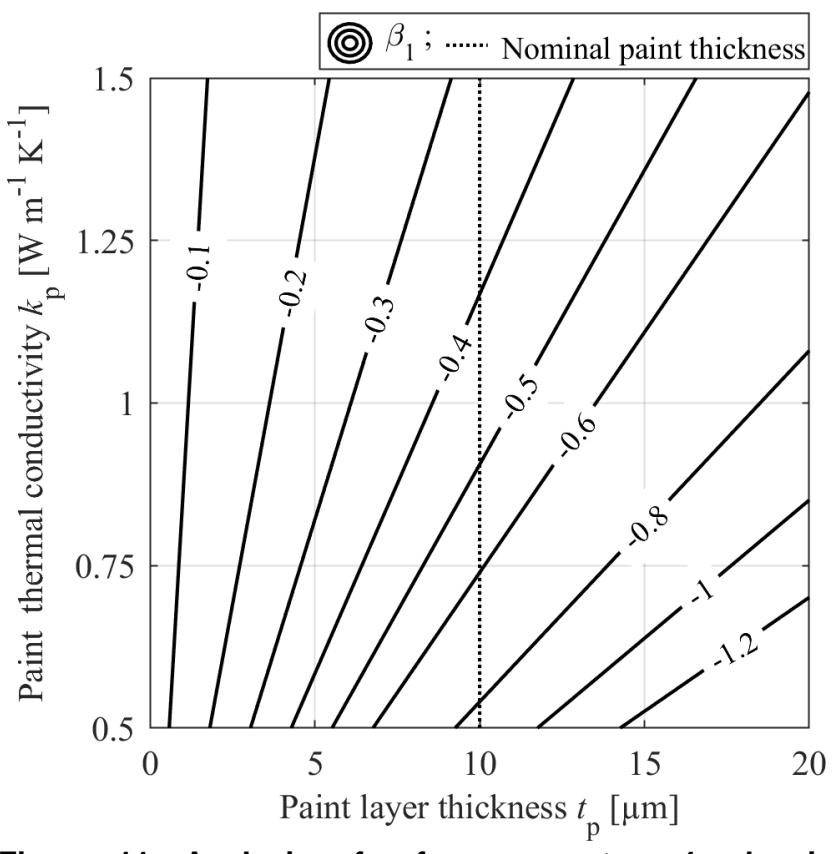

Figure 11: Analysis of reference system 4, showing difference between measured and true external surface temperature as a function of paint thickness and conductivity.

Figure 12 shows the experimental results from reference system 4 in terms of normalized temperature $\phi$ distribution around the exposed copper disk. It is worth mentioning that the absolute levels of $\phi$ differ from Figure 8a because the test were carried on different vanes. However, both measurement systems were approximately at the same location on the vane suction side. Improvements associated to the new system are observed in Figure 12a, where a uniform distribution of normalized temperature is attained on the copper disk. This is emphasized by Figure 12b, which shows the lateral distribution of normalized temperature along the line AA' through the copper disk. A circular region with a diameter of approximately 12 pixels (corresponding to the diameter of the copper disk) is found to perform with substantially uniform normalized temperature $(\phi=$ approximately 0.715 ). This arrangement, therefore, allows reducing the sensitivity to the pixel selection or averaging region, even in the case of uncertainty in the location of the thermocouple junction.
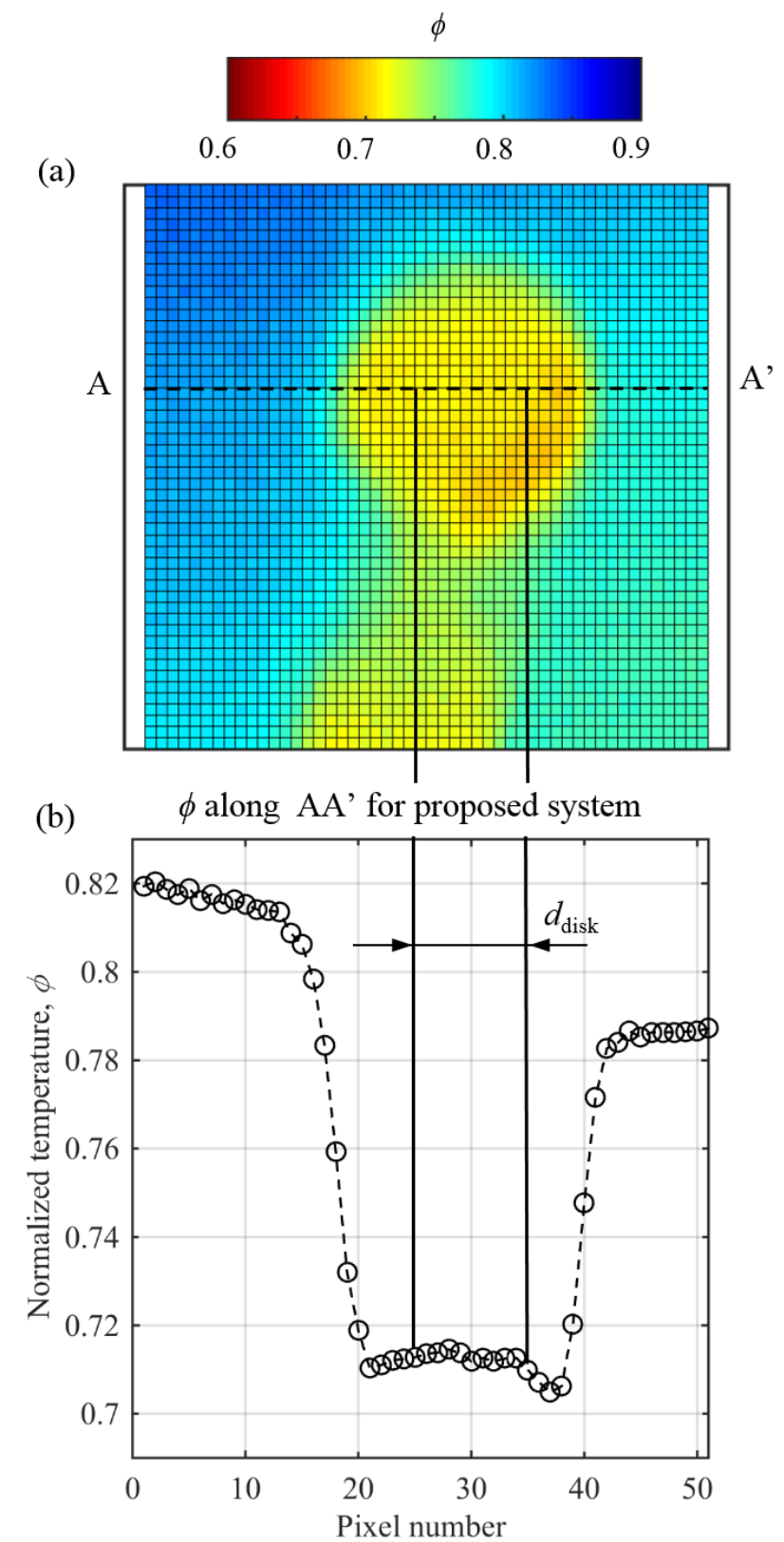

Figure 12: Proposed calibration system (reference system 4, see Figure 7): (a) surface plot of $\phi$ area; (b) line-plot of $\phi$ (along $A^{\prime}$ ) through the thermocouple junction over a 50-by-50 pixel.

\section{CONCLUSIONS}

In this paper, we present a systematic study of the sensitivity of two types of measurement errors $\left(\beta_{1}\right.$ and $\left.\beta_{2}\right)$ to installation conditions seen in typical laboratory IR calibration arrangements, and under realistic conditions of through-wall heat flux. We assess the errors arising from a number of typical in situ IR calibration arrangements by quantifying their sensitivities to the location, thickness and thermal conductivity of the adhesive used to bond the thermocouple to the surface. We define reference system 1 as the case in which the thermocouple is only placed over an adhesive layer; reference system 2 in which the thermocouple is placed over an adhesive layer and overpainted with black paint; reference system 3 in which the thermocouple is in between two adhesive layers. An 
improved system (defined here as reference system 4) aimed to provide a large isothermal region the IR camera FOV, while maintaining a low measurement errors, is also presented and described in detail.

Analysis of system 1 shows a wide range of $\beta_{2}$ error depending on adhesive thickness and thermal conductivity. Errors can be extremely high for common adhesives such as epoxy resin, and relatively low for thin layers of high thermal conductivity adhesives, but such adhesives have low bonding strengths and are not always suitable to adhere a thermocouple on a surface subjected to high-speed flows. It is also noted that system 1 is often impracticable because of the extreme variation in surface emissivity when an overpaint layer is omitted.

Analysis of system 2 shows that paint over the thermocouple increases $\beta_{1}$, and can increase or decrease $\beta_{2}$ depending on the thermal conductivity and thickness of the paint layer with respect to the adhesive layer.

Analysis of system 3 show that an adhesive layer over the thermocouple leads to increased $\beta_{1}$ errors, but that it can reduce $\beta_{2}$ errors. However, the latter is very difficult to achieve in practical applications, as in such thin layers precise thickness control is unrealistic.

Analysis of a proposed new system (system 4) shows that $\beta_{1}$ is primarily dependent on the paint layer thickness and thermal conductivity, and that for typical values of these two parameters encountered in practice, the $\beta_{1}$ can be rendered extremely low by adoption of the proposed scheme. It was also shown that system 4 provides a large isothermal region in the FOV of the IR camera, hence reducing the uncertainty associated with the pixel selection compared to conventional measurement system (system 1 to 3 ).

The work of this paper is developed in response to the lack of data presented in the literature on the effect of typical calibration arrangements on the errors arising in in-situ IR calibrations in environments with high through-wall heat flux and lateral temperature gradient. It is hoped that this paper will present a framework for quantifying such errors in typical calibration arrangements, and that by adoption of the proposed technique error in the calibration process might be substantially reduced.

\section{ACKNOWLEDGMENTS}

The support of Rolls-Royce plc is gratefully acknowledged. The authors would also like to acknowledge the technicians Trevor Godfrey, Gregory King and Dominic Harris for their help in the manufacture of the new measurement system.

\section{NOMENCLATURE}

\section{Roman}

$d \quad$ diameter, $\mathrm{m}$

$h \quad$ heat transfer coefficient, $\mathrm{W} \mathrm{m} \mathrm{m}^{-2} \mathrm{~K}^{-1}$

$k$ thermal conductivity, $\mathrm{W} \mathrm{m}^{-1} \mathrm{~K}^{-1}$

$q \quad$ heat flux, $\mathrm{W} \mathrm{m}^{-2}$

$t \quad$ thickness, $\mathrm{m}$

$T \quad$ temperature, $\mathrm{K}$

\section{Greek}

$\phi \quad$ normalized wall temperature

$\theta \quad$ overall cooling effectiveness

\section{Subcripts}

a adhesive

bb black body

ext external side

int internal side

$\mathrm{p} \quad$ paint

w wall

0 total

$\begin{array}{ll}\text { Abbreviations } \\ \text { FOV } & \text { field of view } \\ \text { HPNGV } & \text { high-pressure nozzle guide vane } \\ \text { HTC } & \text { heat transfer coefficient } \\ \text { IR } & \text { infrared } \\ \text { RTD } & \text { resistance temperature detectors } \\ \text { TC } & \text { thermocouple }\end{array}$

\section{REFERENCES}

Ekkad, S. V., Ou, A. and Rivir, R. (2004). A transient infrared thermography method for simultaneous film cooling effectiveness and heat transfer coefficient measurements from a single test. ASME Journal of Turbomachinery, 126(4), pp. 597-603.

Kirollos, B., Lubbock, R., Beard, P., Goenaga, F., Rawlinson, A., Janke, E. and Povey, T. (2017). ECAT: an engine component aerothermal facility at the university of oxford. ASME paper GT2017-64736, ASME Turbo Expo 2017: Technical Conference and Exposition, Charlotte, USA, June 26-30.

Lee, H. and Neville, K. (1967). Handbook of epoxy resins. New York: McGraw Hill. ISBN 0070369976.

Martiny, M., Schiele, R., Gritsch, A., Schulz, S. and Witting, S. (1996). In situ calibration for quantitative infrared thermography. QIRT'96 Eurotherm Seminar No. 50, Stuttgart, Germany, Sept. 2-5.

Ochs, M., Horbach, T., Schulz, A., Koch, R. and Bauer, H-J. (2009). A novel calibration method for an infrared thermography system applied to heat transfer experiments. Measurement Science and Technology, 20(7), pp. 075103075112.

Raghu, O. and Philip, J. (2006). Thermal properties of paint coatings on different backings using a scanning photo acoustic technique. Measurement Science and Technology, 17(11), pp. 2945-2949.

Schulz, A. (2000). Infrared thermography as applied to film cooling of gas turbine components. Measurement Science and Technology, 11(7), pp. 948-956. 